\title{
Inequalities for dual quermassintegrals of the radial $p$ th mean bodies
}

\section{Weidong Wang* and Ting Zhang}

\section{${ }^{*}$ Correspondence:}

wdwxh722@163.com

Department of Mathematics, China

Three Gorges University, Yichang,

443002, China

\begin{abstract}
Gardner and Zhang defined the notion of radial $p$ th mean body $(p>-1)$ in the Euclidean space $\mathbb{R}^{n}$. In this paper, we obtain inequalities for dual quermassintegrals of the radial pth mean bodies. Further, we establish dual quermassintegrals forms of the Zhang projection inequality and the Rogers-Shephard inequality, respectively. Finally, Shephard's problem concerning the radial $p$ th mean bodies is shown when $p>0$.
\end{abstract} MSC: 52A40; 52A20

Keywords: radial pth mean body; dual quermassintegrals; Zhang projection inequality; Rogers-Shephard inequality

\section{Introduction}

Let $\mathcal{K}^{n}$ denote the set of convex bodies (compact, convex subsets with nonempty interiors) in the Euclidean space $\mathbb{R}^{n}$ for the set of convex bodies containing the origin in their interiors in $\mathbb{R}^{n}$ by $\mathcal{K}_{o}^{n}$. Let $S^{n-1}$ denote the unit sphere in $\mathbb{R}^{n}$, denote by $V(K)$ the $n$-dimensional volume of body $K$ for the standard unit ball $B$ in $\mathbb{R}^{n}$, define $\omega_{n}=V(B)$.

If $K$ is a compact star-shaped (about the origin) in $\mathbb{R}^{n}$, its radial function, $\rho_{K}=\rho(K, \cdot)$, is defined by (see $[1,2])$

$$
\rho(K, u)=\max \{\lambda \geq 0: \lambda u \in K\}
$$

for all $u \in S^{n-1}$. If $\rho_{K}$ is positive and continuous, $K$ will be called a star body (about the origin). Let $\mathcal{S}_{o}^{n}$ denote the set of star bodies (about the origin) in $\mathbb{R}^{n}$. Two star bodies $K$ and $L$ are said to be dilates (of one another) if $\rho_{K}(u) / \rho_{L}(u)$ is independent of $u \in S^{n-1}$.

The notion of radial $p$ th mean body was given by Gardner and Zhang (see [3]). For $K \in \mathcal{K}^{n}$, the radial $p$ th mean body $R_{p} K$ of $K$ is defined for nonzero $p>-1$ by

$$
\rho_{R_{p} K}(u)=\left(\frac{1}{V(K)} \int_{K} \rho_{K}^{p}(x, u) d x\right)^{\frac{1}{p}}
$$

for each $u \in S^{n-1}$; define $R_{0} K$ by

$$
\rho_{R_{0} K}(u)=\exp \left(\frac{1}{V(K)} \int_{K} \log \rho_{K}(x, u) d x\right)
$$

for each $u \in S^{n-1}$.

\section{Springer}

(0) 2014 Wang and Zhang; licensee Springer. This is an Open Access article distributed under the terms of the Creative Commons Attribution License (http://creativecommons.org/licenses/by/2.0), which permits unrestricted use, distribution, and reproduction in any medium, provided the original work is properly cited. 
In [3], Gardner and Zhang showed the following.

Theorem 1.A If $K \in \mathcal{K}^{n},-1<p<q$, then

$$
D K \subseteq c_{n, q} R_{q} K \subseteq c_{n, p} R_{p} K \subseteq n V(K) \Pi^{*} K
$$

in each inclusion equality holds if and only if $K$ is a simplex. Here

$$
c_{n, p}=(n B(p+1, n))^{-\frac{1}{p}}
$$

for nonzero $p>-1, c_{n, 0}=\lim _{p \rightarrow 0}(n B(p+1, n))^{-\frac{1}{p}}$, and $D K$ and $\Pi^{*} K$ denote the difference body and the polar of projection body, respectively.

From Theorem 1.A, Gardner and Zhang [3] again proved the Zhang projection inequality (also see [4]) and the Rogers-Shephard inequality (also see [5]).

Theorem 1.B (Zhang projection inequality) If $K \in \mathcal{K}^{n}$, then

$$
V\left(\Pi^{*} K\right) V(K)^{n-1} \geq \frac{1}{n^{n}}\left(\begin{array}{c}
2 n \\
n
\end{array}\right),
$$

with equality if and only if $K$ is a simplex.

Theorem 1.C (Rogers-Shephard inequality) If $K \in \mathcal{K}^{n}$, then

$$
V(D K) \leq\left(\begin{array}{c}
2 n \\
n
\end{array}\right) V(K)
$$

with equality if and only if $K$ is a simplex.

In this paper, we continuously research the radial $p$ th mean body. First, we establish inequalities for dual quermassintegrals of the radial $p$ th mean body $R_{p} K$ as follows.

Theorem 1.1 If $K \in \mathcal{K}^{n}, p>0$, real $i \neq n$, then there exists $x_{0} \in K$ such that for $i<n-p$ or $i>n$,

$$
\widetilde{W}_{i}\left(R_{p} K\right) \leq \widetilde{W}_{i}\left(K-x_{0}\right)
$$

for $n-p<i<n$

$$
\widetilde{W}_{i}\left(R_{p} K\right) \geq \widetilde{W}_{i}\left(K-x_{0}\right)
$$

In every inequality, equality holds if and only if $R_{p} K=K-x_{0}$. For $i=n-p$, (1.7) (or (1.8)) is identic. Here, $\widetilde{W}_{i}(K)$ denotes the dual quermassintegrals of $K$ which are given by (see [6])

$$
\widetilde{W}_{i}(K)=\frac{1}{n} \int_{S^{n-1}} \rho(K, u)^{n-i} d S(u) .
$$


Obviously, let $i=0$ in (1.9), then

$$
\widetilde{W}_{0}(K)=\frac{1}{n} \int_{S^{n-1}} \rho(K, u)^{n} d S(u)=V(K) .
$$

Let $i=0$ in Theorem 1.1 and notice that $V\left(K-x_{0}\right)=V(K)$, we easily get the following.

Corollary 1.1 If $K \in \mathcal{K}^{n}, p>0$, then for $p<n$,

$$
V\left(R_{p} K\right) \leq V(K)
$$

for $p>n$,

$$
V\left(R_{p} K\right) \geq V(K)
$$

All with equality if and only if $R_{p} K=K$. For $p=n$, above inequalities are identic.

Note that Corollary 1.1 can be found in [7].

As an application of Theorem 1.1, we obtain the following dual quermassintegrals form of the Zhang projection inequality.

Theorem 1.2 If $K \in \mathcal{K}^{n}, p>0$, real $i \neq n$, then there exists $x_{0} \in K$ such that for $n-p \leq i<n$,

$$
\widetilde{W}_{i}\left(\Pi^{*} K\right) \geq\left[\frac{c_{n, p}}{n V(K)}\right]^{n-i} \widetilde{W}_{i}\left(K-x_{0}\right),
$$

with equality for $i=n-p$ if and only if $K$ is a simplex, for $n-p<i<n$ if and only if $K$ is a simplex and $R_{p} K=K-x_{0}$.

Note that the case of $p=n-i$ in (1.11) can be found in [8].

If $p$ is a positive integer in Theorem 1.2, then by (1.4) we get that

$$
\left(c_{n, p}\right)^{n-i}=\left(\begin{array}{c}
n+p \\
n
\end{array}\right)^{\frac{n-i}{p}} .
$$

Hence, we have the following.

Corollary 1.2 If $K \in \mathcal{K}^{n}, p$ is a positive integer, $i$ is any real, if $n-p \leq i<n$, then there exists $x_{0} \in K$ such that

$$
\widetilde{W}_{i}\left(\Pi^{*} K\right) \geq\left[\frac{1}{n V(K)}\right]^{n-i}\left(\begin{array}{c}
n+p \\
n
\end{array}\right)^{\frac{n-i}{p}} \widetilde{W}_{i}\left(K-x_{0}\right)
$$

with equality for $i=n-p$ if and only if $K$ is a simplex, for $n-p<i<n$ if and only if $K$ is a simplex and $R_{p} K=K-x_{0}$.

Let $i=0$ in Corollary 1.2, and together with (1.12) and (1.10), we have the following.

Corollary 1.3 If $K \in \mathcal{K}^{n}, p \geq n$ and $p$ is an integer, then

$$
V\left(\Pi^{*} K\right) V(K)^{n-1} \geq \frac{1}{n^{n}}\left(\begin{array}{c}
n+p \\
n
\end{array}\right)^{\frac{n}{p}}
$$


with equality for $p=n$ if and only if $K$ is a simplex, for $p>n$ if and only if $K$ is a simplex and there exists $x_{0} \in K$ such that $R_{p} K=K-x_{0}$.

Compared to (1.13) and the Zhang projection inequality (1.5), inequality (1.13) may be regarded as a general Zhang projection inequality.

As another application of Theorem 1.1, we obtain the following dual quermassintegrals form of the Rogers-Shephard inequality.

Theorem 1.3 If $K \in \mathcal{K}^{n}, p>0$ and real $i \neq n$, if $i \leq n-p$ or $i>n$, then there exists $x_{0} \in K$ such that

$$
\widetilde{W}_{i}(D K) \leq\left(c_{n, p}\right)^{n-i} \widetilde{W}_{i}\left(K-x_{0}\right)
$$

with equality for $i=n-p$ if and only if $K$ is a simplex, for $i<n-p$ or $i>n$ if and only if $K$ is a simplex and $R_{p} K=K-x_{0}$.

Similarly, if $p$ is a positive integer in Theorem 1.3, then by (1.12) we obtain the following.

Corollary 1.4 If $K \in \mathcal{K}^{n}, p$ is a positive integer, $i$ is any real, if $i \leq n-p$ or $i>n$, then there exists $x_{0} \in K$ such that

$$
\widetilde{W}_{i}(D K) \leq\left(\begin{array}{c}
n+p \\
n
\end{array}\right)^{\frac{n-i}{p}} \widetilde{W}_{i}\left(K-x_{0}\right)
$$

with equality for $i=n-p$ if and only if $K$ is a simplex, for $i<n-p$ or $i>n$ if and only if $K$ is a simplex and $R_{p} K=K-x_{0}$.

Taking $i=0$ in Corollary 1.4, and using (1.12) and (1.10), we get the following.

Corollary 1.5 If $K \in \mathcal{K}^{n}, p$ is a positive integer and $p \leq n$, then

$$
V(D K) \leq\left(\begin{array}{c}
n+p \\
n
\end{array}\right)^{\frac{n}{p}} V(K)
$$

with equality for $p=n$ if and only if $K$ is a simplex, for $p<n$ if and only if $K$ is a simplex and there exists $x_{0} \in K$ such that $R_{p} K=K-x_{0}$.

Compared to (1.15) and the Rogers-Shephard inequality (1.6), inequality (1.15) may be regarded as a general Rogers-Shephard inequality.

In addition, we also give the Shephard-type problem for the radial $p$ th mean bodies in Section 4.

\section{Preliminaries}

\subsection{Support function, difference body and projection body}

If $K \in \mathcal{K}^{n}$, then its support function, $h_{K}=h(K, \cdot)$, is defined by (see $\left.[1,2]\right)$

$$
h(K, x)=\max \{x \cdot y: y \in K\}, \quad x \in \mathbb{R}^{n},
$$

where $x \cdot y$ denotes the standard inner product of $x$ and $y$. 
If $K \in \mathcal{K}_{o}^{n}$, the polar body of $K, K^{*}$, is defined by (see $\left.[1,2]\right)$

$$
K^{*}=\left\{x \in \mathbb{R}^{n}: x \cdot y \leq 1, y \in K\right\} .
$$

If $K \in \mathcal{K}^{n}$, the difference body, $D K=K+(-K)$, of $K$ is defined by (see [1])

$$
h(D K, u)=h(K, u)+h(K,-u)
$$

for all $u \in S^{n-1}$.

For $K \in \mathcal{K}^{n}$, the projection body of $K, \Pi K$, is a centered convex body whose support function is given by (see [1])

$$
h(\Pi K, u)=V_{n-1}\left(K \mid u^{\perp}\right)
$$

for all $u \in S^{n-1}$, where $V_{n-1}$ denotes $(n-1)$-dimensional volume, and $K \mid u^{\perp}$ denotes the image of orthogonal projection of $K$ onto the codimensional 1 subspace orthogonal to $u$.

\subsection{Extended radial function}

If $K$ is compact star-shaped with respect to $x \in \mathbb{R}^{n}$, its radial function $\rho_{K}(x, \cdot)$ with respect to $x$ is defined, for all $u \in S^{n-1}$ such that the line through $x$ parallel to $u$ intersects $K$, by (see [3])

$$
\rho_{K}(x, u)=\max \{\lambda \geq 0: x+\lambda u \in K\} .
$$

From (1.1) and (2.1), we easily know that

$$
\rho_{K}(x, u)=\rho_{K-x}(u)
$$

for all $u \in S^{n-1}$. We call $\rho_{K}(x, \cdot)$ the extended radial function of $K$ with respect to $x$. If $x$ is the origin $o$, then $\rho_{K}(x, u)=\rho_{K}(u)$ for all $u \in S^{n-1}$.

From (2.2) and (1.2), obviously,

$$
\rho_{R_{p} K}(u)=\left(\frac{1}{V(K)} \int_{K} \rho_{K-x}^{p}(u) d x\right)^{\frac{1}{p}} .
$$

\section{3 $L_{p}$-Dual mixed quermassintegrals}

If $K, L \in \mathcal{S}_{o}^{n}, p>0, \lambda, \mu \geq 0$ (not both zero), the $L_{p}$-radial combination, $\lambda \cdot K \tilde{+}_{p} \mu \cdot L \in \mathcal{S}_{o}^{n}$, of $K$ and $L$ is defined by (see $[9,10])$

$$
\rho\left(\lambda \cdot K \tilde{+}_{p} \mu \cdot L, \cdot\right)^{p}=\lambda \rho(K, \cdot)^{p}+\mu \rho(L, \cdot)^{p} .
$$

Associated with (2.4) and (1.9), we define a class of $L_{p}$-dual mixed quermassintegrals as follows: For $K, L \in \mathcal{S}_{o}^{n}, p>0, \varepsilon>0$ and real $i \neq n$, the $L_{p}$-dual mixed quermassintegrals, $\widetilde{W}_{p, i}(K, L)$, of $K$ and $L$ are defined by

$$
\frac{n-i}{p} \widetilde{W}_{p, i}(K, L)=\lim _{\varepsilon \rightarrow 0^{+}} \frac{\widetilde{W}_{i}\left(K \tilde{+}_{p} \varepsilon \cdot L\right)-\widetilde{W}_{i}(K)}{\varepsilon} .
$$


Let $i=0$ in definition (2.5), and together with (1.10), we write that $\widetilde{W}_{p, 0}(K, L)=\widetilde{V}_{p}(K, L)$, then

$$
\frac{n}{p} \widetilde{V}_{p}(K, L)=\lim _{\varepsilon \rightarrow 0^{+}} \frac{V\left(K \tilde{+}_{p} \varepsilon \cdot L\right)-V(K)}{\varepsilon} .
$$

Here $\widetilde{V}_{p}(K, L)$ denotes a type of $L_{p}$-dual mixed volume of $K$ and $L$ which is defined in [9, 11] (for $p \geq 1$ also see [12]).

From definition (2.5), the integral representation of $L_{p}$-dual mixed quermassintegrals can be established as follows.

Theorem 2.1 If $K, L \in \mathcal{S}_{o}^{n}, p>0$, and real $i \neq n$, then

$$
\widetilde{W}_{p, i}(K, L)=\frac{1}{n} \int_{S^{n-1}} \rho_{K}^{n-p-i}(u) \rho_{L}^{p}(u) d S(u) .
$$

Proof From (2.4) and (2.5), for $i \neq n$, we have that

$$
\begin{aligned}
\lim _{\varepsilon \rightarrow 0^{+}} & \frac{\widetilde{W}_{i}\left(K \hat{+}_{p} \varepsilon \cdot L\right)-\widetilde{W}_{i}(K)}{\varepsilon} \\
= & \lim _{\varepsilon \rightarrow 0^{+}} \frac{1}{n} \int_{S^{n-1}} \frac{\rho\left(K \hat{+}_{p} \varepsilon \cdot L, u\right)^{n-i}-\rho(K, u)^{n-i}}{\varepsilon} d S(u) \\
\quad= & \lim _{\varepsilon \rightarrow 0^{+}} \frac{1}{n} \int_{S^{n-1}} \frac{\left[\rho(K, u)^{p}+\varepsilon \rho(L, u)^{p}\right]^{\frac{n-i}{p}}-\rho(K, u)^{n-i}}{\varepsilon} d S(u) .
\end{aligned}
$$

By Hospital's rule we see that

$$
\begin{aligned}
\lim _{\varepsilon \rightarrow 0^{+}} & {\left[\rho(K, \cdot)^{p}+\varepsilon \rho(L, \cdot)^{p}\right]^{\frac{n-i}{p}}-\rho(K, \cdot)^{n-i} } \\
\varepsilon & =\lim _{\varepsilon \rightarrow 0^{+}} \rho_{K}^{n-i} \frac{\left[1+\varepsilon\left(\rho_{K} / \rho_{L}\right)^{p}\right]^{\frac{n-i}{p}}-1}{\varepsilon} \\
= & \frac{n-i}{p} \rho_{K}^{n-p-i} \rho_{L}^{p},
\end{aligned}
$$

thus we get formula (2.6) by definition (2.5).

From (2.6), we easily know that

$$
\begin{aligned}
& \widetilde{W}_{p, i}(K, K)=\frac{1}{n} \int_{S^{n-1}} \rho(K, u)^{n-i} d S(u)=\widetilde{W}_{i}(K), \\
& \widetilde{W}_{p, n-p}(K, L)=\widetilde{W}_{n-p}(L) .
\end{aligned}
$$

The Minkowski inequality for the $L_{p}$-dual mixed quermassintegrals is given as follows.

Theorem 2.2 Let $K, L \in \mathcal{S}_{o}^{n}, p>0$, and real $i \neq n$, then for $i<n-p$,

$$
\widetilde{W}_{p, i}(K, L) \leq \widetilde{W}_{i}(K)^{(n-p-i) /(n-i)} \widetilde{W}_{i}(L)^{p /(n-i)} ;
$$


for $n-p<i<n$ or $i>n$,

$$
\widetilde{W}_{p, i}(K, L) \geq \widetilde{W}_{i}(K)^{(n-p-i) /(n-i)} \widetilde{W}_{i}(L)^{p /(n-i)} .
$$

In every inequality, equality holds if and only if $K$ and $L$ are dilates. For $i=n-p$, (2.9) (or (2.10)) is identic.

Proof For $i<n-p$, from (2.6) and together with the Hölder inequality (see [13]), we have that

$$
\begin{aligned}
\widetilde{W}_{p, i}(K, L) & =\frac{1}{n} \int_{S^{n-1}} \rho_{K}^{n-p-i}(u) \rho_{L}^{p}(u) d S(u) \\
& \leq\left[\frac{1}{n} \int_{S^{n-1}}\left[\rho_{K}^{n-p-i}(u)\right]^{\frac{n-i}{n-p-i}} d S(u)\right]^{\frac{n-p-i}{n-i}}\left[\frac{1}{n} \int_{S^{n-1}}\left[\rho_{L}^{p}(u)\right]^{\frac{n-i}{p}} d S(u)\right]^{\frac{p}{n-i}} \\
& =\left[\frac{1}{n} \int_{S^{n-1}} \rho_{K}^{n-i}(u) d S(u)\right]^{\frac{n-p-i}{n-i}}\left[\frac{1}{n} \int_{S^{n-1}} \rho_{L}^{n-i}(u) d S(u)\right]^{\frac{p}{n-i}} \\
& =\widetilde{W}_{i}(K)^{\frac{n-p-i}{n-i}} \widetilde{W}_{i}(L)^{\frac{p}{n-i}}
\end{aligned}
$$

which gives inequality (2.9) when $i<n-p$. According to the condition that equality holds for the Hölder inequality, we know that the equality holds in inequality (2.9) if and only if $K$ and $L$ are dilates.

Similarly, we can prove for $n-p<i<n$ or $i>n$, inequality (2.10) is true.

For $i=n-p$, by (2.8) and (2.3) then

$$
\widetilde{W}_{p, i}(K, L)^{n-i}=\widetilde{W}_{p, n-p}(K, L)^{p}=\widetilde{W}_{n-p}(L)^{p}
$$

and

$$
\widetilde{W}_{i}(K)^{n-p-i} \widetilde{W}_{i}(L)^{p}=\widetilde{W}_{n-p}(K)^{n-p-i} \widetilde{W}_{n-p}(L)^{p}=\widetilde{W}_{n-p}(L)^{p},
$$

thus (2.9) (or (2.10)) is identic when $i=n-p$.

\section{Proofs of the theorems}

The proofs of the theorems require the following lemma.

Lemma 3.1 If $K \in \mathcal{K}^{n}, p>0$, and real $i \neq n$, then for any $Q \in \mathcal{S}_{o}^{n}$,

$$
\widetilde{W}_{p, i}\left(Q, R_{p} K\right)=\frac{1}{V(K)} \int_{K} \widetilde{W}_{p, i}(Q, K-x) d x .
$$

Proof Using (2.6) and (2.3), then for any $Q \in \mathcal{S}_{o}^{n}$, we have that

$$
\begin{aligned}
\widetilde{W}_{p, i}\left(Q, R_{p} K\right) & =\frac{1}{n} \int_{S^{n-1}} \rho(Q, u)^{n-p-i} \rho\left(R_{p} K, u\right)^{p} d S(u) \\
& =\frac{1}{n V(K)} \int_{S^{n-1}} \int_{K} \rho(Q, u)^{n-p-i} \rho(K-x, u)^{p} d x d S(u) \\
& =\frac{1}{V(K)} \int_{K} \widetilde{W}_{p, i}(Q, K-x) d x .
\end{aligned}
$$


Proof of Theorem 1.1 For $i<n-p$, let $Q=R_{p} K$ in (3.1), this together with (2.6), (2.7) and (2.9) gives

$$
\begin{aligned}
\widetilde{W}_{i}\left(R_{p} K\right) & =\frac{1}{V(K)} \int_{K} \widetilde{W}_{p, i}\left(R_{p} K, K-x\right) d x \\
& \leq \frac{1}{V(K)} \int_{K} \widetilde{W}_{i}\left(R_{p} K\right)^{\frac{n-p-i}{n-i}} \widetilde{W}_{i}(K-x)^{\frac{p}{n-i}} d x \\
& =\frac{1}{V(K)} \widetilde{W}_{i}\left(R_{p} K\right)^{\frac{n-p-i}{n-i}} \int_{K} \widetilde{W}_{i}(K-x)^{\frac{p}{n-i}} d x,
\end{aligned}
$$

i.e.,

$$
\widetilde{W}_{i}\left(R_{p} K\right)^{\frac{p}{n-i}} \leq \frac{1}{V(K)} \int_{K} \widetilde{W}_{i}(K-x)^{\frac{p}{n-i}} d x .
$$

Therefore, according to the integral mean value theorem, there exists $x_{0} \in K$ such that

$$
\widetilde{W}_{i}\left(R_{p} K\right)^{\frac{p}{n-i}} \leq \frac{1}{V(K)} \widetilde{W}_{i}\left(K-x_{0}\right)^{\frac{p}{n-i}} \int_{K} d x=\widetilde{W}_{i}\left(K-x_{0}\right)^{\frac{p}{n-i}} .
$$

Since $p>0$ and $i<n-p$, thus we get inequality (1.7). According to the condition that equality holds in inequality (2.9), we see that with equality in (1.7) if and only if $R_{p} K$ and $K-x_{0}$ are dilates. This combined with (1.7), we know that equality holds in (1.7) if and only if $R_{p} K=K-x_{0}$.

Similarly, for $n-p<i<n$ or $i>n$, from inequality (2.10) and equality (3.1), then

$$
\widetilde{W}_{i}\left(R_{p} K\right)^{\frac{p}{n-i}} \geq \widetilde{W}_{i}\left(K-x_{0}\right)^{\frac{p}{n-i}} .
$$

Hence, we have that for $i>n$ and $p>0$,

$$
\widetilde{W}_{i}\left(R_{p} K\right) \leq \widetilde{W}_{i}\left(K-x_{0}\right)
$$

for $n-p<i<n$ and $p>0$,

$$
\widetilde{W}_{i}\left(R_{p} K\right) \geq \widetilde{W}_{i}\left(K-x_{0}\right) .
$$

From this, we get inequality (1.7) and inequality (1.8), respectively, and equality holds in the above inequalities if and only if $R_{p} K=K-x_{0}$.

For $i=n-p$, by (2.8) and (3.1) we see that (1.7) (or (1.8)) is identic.

Proof of Theorem 1.2 From (1.3), we have that $c_{n, p} R_{p} K \subseteq n V(K) \Pi^{*} K$ for $p>-1$, then

$$
\left(c_{n, p}\right)^{n-i} \widetilde{W}_{i}\left(R_{p} K\right) \leq(n V(K))^{n-i} \widetilde{W}_{i}\left(\Pi^{*} K\right),
$$

with equality if and only if $K$ is a simplex. Hence, together with (1.8), then for $n-p<i<n$ and $p>0$, we obtain that

$$
\widetilde{W}_{i}\left(\Pi^{*} K\right) \geq\left[\frac{c_{n, p}}{n V(K)}\right]^{n-i} \widetilde{W}_{i}\left(K-x_{0}\right),
$$

which is desired (1.11). 
Associated with the cases of equality holding in (3.2) and (1.8), we see that equality holds in (1.11) for $i=n-p$ if and only if $K$ is a simplex, for $n-p<i<n$ if and only if $K$ is a simplex and $R_{p} K=K-x_{0}$.

Proof of Theorem 1.3 From (1.3), we know that $D K \subseteq c_{n, p} R_{p} K$ for $p>-1$, thus

$$
\widetilde{W}_{i}(D K) \leq\left(c_{n, p}\right)^{n-i} \widetilde{W}_{i}\left(R_{p} K\right)
$$

with equality if and only if $K$ is a simplex. Hence, together with (1.7), then for $p>0, i<n-p$ or $i>n$, we get that

$$
\widetilde{W}_{i}(D K) \leq\left(c_{n, p}\right)^{n-i} \widetilde{W}_{i}\left(K-x_{0}\right)
$$

this is just (1.14).

Combining with the cases of equality holding in (3.3) and (1.7), we see that equality holds in (1.14) for $i=n-p$ if and only if $K$ is a simplex, for $i<n-p$ or $i>n$ if and only if $K$ is a simplex and $R_{p} K=K-x_{0}$.

\section{Shephard-type problem}

In this section, we research the Shephard-type problem for the radial $p$ th mean bodies. Recall that Zhou and Wang in [7] gave the Shephard-type problem for the radial $p$ th mean bodies as follows.

Theorem 4.A Let $K, L \in \mathcal{K}^{n}, p>0$, if $R_{p} K \subseteq R_{p} L$, then

$$
V(K) \leq V(L)
$$

with equality if and only if $R_{p} K=R_{p} L$ and $K$ is a translation of $L$.

Here, we obtain a stronger result for the Shephard-type problem of the radial $p$ th mean bodies. Our result is the following theorem.

Theorem 4.1 Let $K, L \in \mathcal{K}^{n}, p>0$, if $R_{p} K \subseteq R_{p} L$, then there exist $x_{0} \in K$ and $y_{0} \in L$ such that

$$
K-x_{0} \subseteq L-y_{0}
$$

with equality if and only if $R_{p} K=R_{p} L$ and $K-x_{0}=L-y_{0}$.

Proof Since $R_{p} K \subseteq R_{p} L$ for $p>0$, thus $\rho_{R_{p} K}^{p}(u) \leq \rho_{R_{p} L}^{p}(u)$ for all $u \in S^{n-1}$, i.e.,

$$
\frac{1}{V(K)} \int_{K} \rho_{K-x}^{p}(u) d x \leq \frac{1}{V(L)} \int_{L} \rho_{L-y}^{p}(u) d y .
$$

Therefore, by the integral mean value theorem, there exist $x_{0} \in K$ and $y_{0} \in L$ such that

$$
\rho_{K-x_{0}}^{p}(u) \frac{1}{V(K)} \int_{K} d x \leq \rho_{L-y_{0}}^{p}(u) \frac{1}{V(L)} \int_{L} d y,
$$


thus

$$
\rho_{K-x_{0}}^{p}(u) \leq \rho_{L-y_{0}}^{p}(u)
$$

for all $u \in S^{n-1}$. This yields (4.1).

\section{Competing interests}

The authors declare that they have no competing interests.

\section{Authors' contributions}

All authors contributed equally to the writing of this paper. All authors read and approved the final manuscript.

\section{Acknowledgements}

Research is supported in part by the Natural Science Foundation of China (Grant No. 11371224) and Innovation Foundation of Graduate Student of China Three Gorges University (Grant No. 2014CX097).

Received: 8 January 2014 Accepted: 26 June 2014 Published: 22 July 2014

\section{References}

1. Gardner, RJ: Geometric Tomography, 2nd edn. Cambridge University Press, Cambridge (2006)

2. Schneider, R: Convex Bodies: The Brunn-Minkowski Theory, 2nd edn. Cambridge University Press, Cambridge (2014)

3. Gardner, RJ, Zhang, GY: Affine inequalities and radial mean bodies. Am. J. Math. 120, 505-528 (1998)

4. Zhang, GY: Restricted chord projection and affine inequalities. Geom. Dedic. 39, 213-222 (1991)

5. Rogers, CA, Shephard, GC: The difference body of a convex body. Arch. Math. 8, 220-233 (1957)

6. Lutwak, E: Dual mixed volumes. Pac. J. Math. 58, 531-538 (1975)

7. Zhou, YP, Wang, WD: Inequalities for the radial p-th mean bodies. Wuhan Univ. J. Nat. Sci. 17(4), 1-5 (2012)

8. Wang, WD: On extensions of the Zhang's projection inequality. Adv. Appl. Math. Sci. 2, $199-207$ (2010)

9. Haberl, C: $L_{p}$ Intersection bodies. Adv. Math. 217(6), 2599-2624 (2008)

10. Haberl, C, Ludwig, M: A characterization of $L_{p}$ intersection bodies. Int. Math. Res. Not. 2006, Article ID 10548 (2006)

11. Yuan, J, Cheung, W-S: L. -Intersection bodies. J. Math. Anal. Appl. 339(2), 1431-1439 (2008)

12. Yu, WY, Wu, DH, Leng, GS: Quasi $L_{p}$-intersection bodies. Acta Math. Sin. 23(11), 1937-1948 (2007)

13. Hardy, GH, Littlewood, JE, Pólya, G: Inequalities. Cambridge University Press, Cambridge (1959)

\section{Submit your manuscript to a SpringerOpen ${ }^{\circ}$ journal and benefit from:}

- Convenient online submission

Rigorous peer review

- Immediate publication on acceptance

- Open access: articles freely available online

- High visibility within the field

- Retaining the copyright to your article 